\title{
Significados e fatores influenciadores da pesquisa em enfermagem no Hospital Universitário Walter Cantídio*
}

\author{
MEANINGS AND FACTORS THAT INFLUENCE NURSING RESEARCH ATTHE \\ WALTER CANTÍDIOUNIVERSITYHOSPITAL
}

\section{SIGNIFICADOS Y FACTORES INFLUENCIABLES DE LA PESQUISA EN ENFERMERÍA EN EL HOSPITAL UNIVERSITARIO WALTER CANTÍDIO}

\section{Ana Maria Ribeiro Cardoso Mesquita', Wagner Bandeira Andriola", Neiva Francinely Cunha Vieira"', , Vicente de Paulo Mesquitalv}

\section{RESUMO}

Este trabalho procurou analisar os fatores que interferem na pesquisa em Enfermagem, na visão dos enfermeiros de um hospital público universitário na cidade de Fortaleza-CE. Estudo de natureza quanti-qualitativa, realizado com 22 enfermeiros, cujos dados foram colhidos no período de $04 / 03$ a 30/04 de 2005. Os resultados destacam o gosto pela pesquisa e sua importância na melhora da práxis, crescimento pessoal e profissional, significando atividade que produz saber, que traz soluções para o cotidiano, mas que pressupõe um investimento pessoal muito grande, ensejado pela carência de recursos humanos, físicos, materiais e por lacunas na formação acadêmica, alertando para a necessidade de mudança curricular na graduação. Portanto, os enfermeiros consideram a realização de pesquisa um ganho pessoal e institucional, mas que depende apenas de investimento e esforço próprios, tornando-se uma atividade laboriosa, desmotivadora, que necessita de uma política organizacional neste aspecto.

\section{DESCRITORES}

Pesquisa em enfermagem. Hospitais Universitários. Educação continuada em Enfermagem.

\section{ABSTRACT}

This study analyzed the meanings and factors that may interfere in Nursing research in the point of view of assistant nurses working in a public University Hospital in the city of Fortaleza, State of Ceará. A quantitative-qualitative survey was carried out with 22 nurses between March 4 and April 30,2005 . The findings pointed out that nurses value the importance of research for enriching their profession. However, although research produces knowledge and solves problems that occur in their daily activities, it demands heavy investment from professionals who are overburdened with work in an environment lacking human, physical and material conditions and often have inadequate academic formation, which points out to the need for improving research skills in Nursing courses. In conclusion, nurses consider research rewarding professionally and for self-development, but it depends exclusively on the individuals' effort and thus become a painstaking, unmotivating activity that requires an organizational policy to be enhanced.

\section{KEY WORDS}

Nursing research.

Hospitals, University.

Education, nursing, continuing.

\section{RESUMEN}

Este trabajo buscó analizar los factores que interfieren en la pesquisa en Enfermería, en la visión de los enfermeros de un hospital público universitario en la ciudad de Fortaleza, Estado del Ceará. Estudio de naturaleza volcada en la cantidad y calidad, realizado con 22 enfermeros, cuyos datos fueron recogidos en el periodo de 04/03 a 30/04 de 2005. Los resultados destacan el gusto por la pesquisa y su importancia en la mejora de la praxis, crecimiento personal y profesional, significando actividad que produce saber, que trae soluciones para el cotidiano, más que presupone una inversión personal muy grande, ofrecido por la carencia de recursos humanos, físicos, materiales y por los huecos en la formación académica, alertando para la necesidad de cambios curriculares en la gradación. Por lo tanto, los enfermeros consideran la realización de la pesquisa un gaño personal e institucional, más que depende apenas de inversión y esfuerzo propios, tornándose una actividad laboriosa, desmotivadota, que necesita de una política organizacional en este aspecto.

\section{DESCRIPTORES}

Investigación en enfermería. Hospitales Universitarios. Educación continua en enfermería.

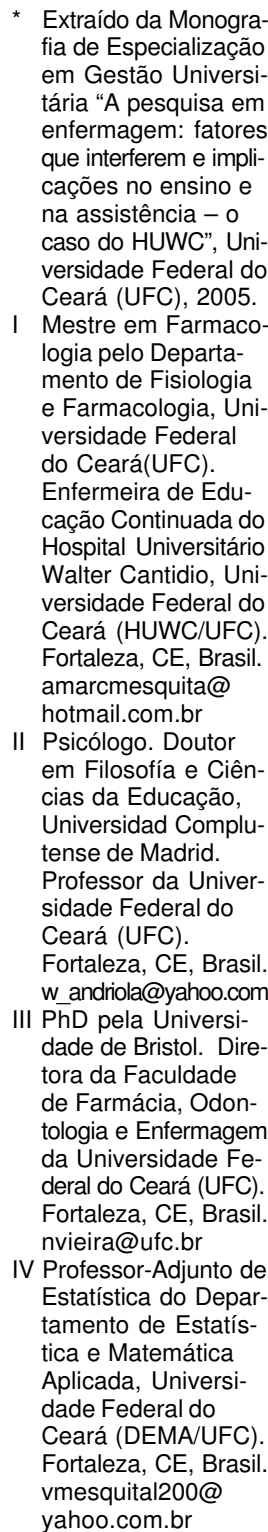

Extraído da Monografia de Especialização em Gestão Universitária "A pesquisa em enfermagem: fatores que interferem e implicações no ensino e na assistência - o caso do HUWC", Universidade Federal do Ceará (UFC), 2005.

I Mestre em Farmacologia pelo Departamento de Fisiologia e Farmacologia, Universidade Federal do Ceará(UFC). Enfermeira de Educação Continuada do Hospital Universitário Walter Cantidio, Universidade Federal do Ceará (HUWC/UFC). Fortaleza, CE, Brasil. amarcmesquita@ hotmail.com.br

II Psicólogo. Doutor em Filosofía e Ciências da Educação, Universidad Complutense de Madrid. Professor da Universidade Federal do Ceará (UFC). Fortaleza, CE, Brasil. w_andriola@yahoo.com III PhD pela Universidade de Bristol. Diretora da Faculdade de Farmácia, Odontologia e Enfermagem da Universidade $\mathrm{Fe}$ deral do Ceará (UFC) Fortaleza, CE, Brasil. nvieira@ufc.br

IV Professor-Adjunto de Estatística do Departamento de Estatística e Matemática Aplicada, Universidade Federal do Ceará (DEMA/UFC). Fortaleza, CE, Brasil. vmesquital200@ yahoo.com.br 


\section{INTRODUÇÃO}

\section{Do problema ao tema}

O Hospital Universitário Walter Cantídio, da Universidade Federal do Ceará (HUWC/UFC), é uma instituição de ensino que desenvolve ações de complexidade distinta no atendimento aos usuários, dependendo da conjugação do trabalho de vários profissionais. Essa missão de assistir e ensinar exige um quadro de pessoal de Enfermagem, quantitativa e qualitativamente, que integre em suas áreas de competência as atividades de ensino, pesquisa e de assistência. Acreditamos ainda que, por ser um hospital de referência acadêmica deve abraçar a visão da integralidade da cultura humanística e científica na geração de conhecimentos e no cuidar da saúde de seus usuários.

Teoricamente os hospitais universitários deveriam ser um ambiente privilegiado, não só para a realização de investigações, mas funcionar como centros de desenvolvimento, que estabelecem novas formas e maneiras de pensar ${ }^{(1)}$.

Considerando que os enfermeiros constituem número significativo de profissionais, e que, com sua equipe, formam o maior contingente de recursos humanos no cuidados aos clientes, é oportuno indagar, pela natureza da instituição, a visão dos enfermeiros como geradores e consumidores de conhecimentos, melhorando a qualidade do cuidado de Enfermagem. Pois, pesquisa significa diálogo crítico e criativo com a realidade, culminando na elaboração própria do conhecimento e na capacidade de intervenção ${ }^{(2)}$.

Este estudo teve como objetivos identificar o significado e os fatores que interferem na pesquisa em Enfermagem em um hospital público universitário. Sua justificativa dá-se pela necessidade de conhecer esta realidade nesta instituição de ensino, pois assim poderemos, a partir deste conhecimento, criar condições para fortalecer e unir teoria e prática, contribuindo para a produção de conhecimentos na Enfermagem.

\section{Importância e origem da pesquisa em enfermagem}

A origem da Enfermagem é, freqüentemente, associada ao Cristianismo, à imagem de mulher devotada ao atendimento de seus semelhantes que sofrem, e se consolida com a religiosidade cristã, mantendo-se assim por muitos séculos, até que a cultura ocidental, transformada pelo cientificismo, foi substituindo os dogmas e as crenças pelo conhecimento que o homem adquire mediante a pesquisa, a cognição e a correlação de saberes ${ }^{(3)}$.

Avançando nos tempos, por volta de 1860, surge a Enfermagem Moderna, na Inglaterra, com Florence Nighthingale, criadora de um sistema que serve de base para uma nova forma de fazer e pensar Enfermagem, que possui, entre outras características, uma atuação técnica a partir de princípios científicos. Com base nos dados e observações de sua práti- ca, ela tornou-se a precursora da investigação em Enfermagem, a partir das ações que implementou. Desde então, percebe-se a trajetória da pesquisa como importante recurso no processo de trabalho da Enfermagem, a qual é normatizada por determinantes sociais, políticos e econômicos. A pesquisa em Enfermagem legitima sua ação, busca novas formas de cuidar, aproxima as dimensões teóricas e práticas do trabalho de Enfermagem, contribui para a qualidade de vida da população, produz o saber substantivo e dá sustentação à prática da atividade ${ }^{(4)}$.

$\mathrm{Na}$ literatura científica a pesquisa é entendida como um ato de cuidar que procura responder aos desafios do cotidiano e que contribui para a constituição de uma disciplina ou ciência da Enfermagem ${ }^{(5-6)}$.

Os enfermeiros que participam de pesquisa, portanto, estão auxiliando a desenvolver, aprimorar e ampliar a base científica do conhecimento, que é fundamental ao exercício da Enfermagem.

\section{MÉTODO}

\section{Tipo de estudo}

Estudo de natureza quanti-qualitativa, pois sua abordagem permite abordar o mundo dos significados das ações e relações humanas ${ }^{(7)}$; exploratório e descritivo, porquanto permite compreender determinada realidade e fornece subsídios para a intervenção(8)

\section{Campo de estudo}

Os locais de estudo foram unidades de internações clínicas e cirúrgicas do Hospital Universitário Walter Cantídio, que é um complexo hospitalar universitário e público da cidade de Fortaleza - Ceará - Brasil, pertencente à Universidade Federal do Ceará.

\section{Sujeitos de investigação}

O universo foi constituído pelos enfermeiros de unidades clínicas e cirúrgicas. Os critérios de inclusão na pesquisa foi o tempo de serviço na instituição de pelo menos dois anos, ser chefe de unidade e/ou ter concluído monografia e/ ou publicações nos últimos cinco anos, e aceitar participar do estudo. Estes critérios atendem aos pré-requisitos para a pesquisa qualitativa, na qual as características dos informantes para o que se quer compreender sobre o problema da investigação é o mais importante ${ }^{(9)}$.

\section{Instrumento de coleta de dados}

O instrumento de coleta de dados foi um roteiro de entrevista previamente estruturado, sobre dados sociodemo-gráficos, nível de satisfação com a profissão, cotidiano do en- 
fermeiro e políticas internas relacionadas ao objeto de investigação. Entre as diversas formas de abordagem técnica do trabalho de campo, a entrevista é a mais usual, pois, por seu intermédio, o pesquisador obtém informes contido na fala dos atores sociais acerca do tema investigado ${ }^{(9)}$.

\section{Coleta de dados}

A coleta de dados foi efetivada após a aprovação do projeto pelas autoridades competentes do HUWC e pelo Comitê de Ética em Pesquisa do Complexo Hospitalar da Univer sidade Federal do Ceará (COMEPE) em 04/03/2005, com base a Resolução no 196/96 do Ministério da Saúde ${ }^{(10)}$, que regula pesquisas com seres humanos.

\section{Organização e análise dos dados}

Os dados foram submetidos à análise de conteúdo( ${ }^{(8)}$. Numa etapa posterior, os dados foram analisados, mediante estatística descritiva.

\section{RESULTADOS E DISCUSSÃO}

\section{Descrição dos sujeitos de investigação}

Os dados sociodemográficos e características referentes a formação acadêmica e satisfação profissional dos enfermeiros estão apresentados na Tabela 1.

Tabela 1 - Distribuição das características dos enfermeiros, HUWC/UFC - Fortaleza - 2005

\begin{tabular}{|c|c|c|}
\hline Variáveis Sóciodemográficas e Nível de satisfação profissional & $\mathrm{n}=22$ & $\%$ \\
\hline \multicolumn{3}{|l|}{ SEXO } \\
\hline Masculino & 04 & 18,18 \\
\hline Feminino & 18 & 81,82 \\
\hline TOTAL & 22 & 100,00 \\
\hline \multicolumn{3}{|l|}{ IDADE } \\
\hline $30,0-34,2$ & 07 & 31,82 \\
\hline $34,2-38,4$ & 02 & 09,09 \\
\hline $38,4-42,6$ & 07 & 31,82 \\
\hline $42,6-46,8$ & 03 & 13,64 \\
\hline $46,8-51,0$ & 03 & 13,64 \\
\hline TOTAL & 22 & 100,00 \\
\hline \multicolumn{3}{|l|}{ TEMPO DE SERVIÇO (anos) } \\
\hline $02-06$ & 04 & 18,18 \\
\hline $06-10$ & 06 & 27,27 \\
\hline $10-14$ & 03 & 13,64 \\
\hline $14-18$ & 04 & 18,18 \\
\hline $18-22$ & 05 & 22,73 \\
\hline TOTAL & 22 & 100,00 \\
\hline \multicolumn{3}{|l|}{ INSTITUIÇÃO FORMADORA } \\
\hline UFC (Universidade Federal do Ceará) & 10 & 45,45 \\
\hline UECE (Universidade Estadual do Ceará) & 07 & 31,82 \\
\hline UNIFOR (Universidade de Fortaleza) & 04 & 18,18 \\
\hline Universidade Estadual de Santa Cruz - BA & 01 & 04,55 \\
\hline TOTAL & 22 & 100,00 \\
\hline \multicolumn{3}{|l|}{ TITULAÇĀO } \\
\hline Graduação & 02 & 09,09 \\
\hline Especialização & 17 & 77,27 \\
\hline Mestrado & 03 & 13,64 \\
\hline TOTAL & 22 & 100,00 \\
\hline \multicolumn{3}{|l|}{ CARGO } \\
\hline Chefia & 11 & 50,00 \\
\hline Enfermeiro Clínico & 11 & 50,00 \\
\hline TOTAL & 22 & 100,00 \\
\hline \multicolumn{3}{|l|}{$\mathrm{N}^{\circ}$ DE EMPREGOS } \\
\hline 01 Emprego & 07 & 31,82 \\
\hline 02 Empregos & 12 & 54,55 \\
\hline 03 Empregos & 03 & 13,64 \\
\hline TOTAL & 22 & 100,00 \\
\hline \multicolumn{3}{|l|}{ SATISFAÇAŌ PROFISSIONAL (escala de 0 - 10) } \\
\hline 10 & 02 & 09,09 \\
\hline 09 & 03 & 13,64 \\
\hline 08 & 11 & 50,00 \\
\hline 07 & 06 & 27,27 \\
\hline TOTAL & 22 & 100,00 \\
\hline
\end{tabular}


Conforme os dados da Tabela 1, a maioria dos enfermeiros é do sexo feminino, como era de se esperar, por ser a Enfermagem uma profissão ainda predominantemente feminina. A média de idade foi de 38,68 anos, com desvio - padrão (DP) de 6,04 e coeficiente de variação de Pearson (CVp) $15,61 \%$. As faixas etárias predominantes foram as de 30 a 34,2 anos $(31,82 \%)$ e de 38,4 a 42,6 anos $(31,82 \%)$. A média de tempo de serviço foi de 11,41 anos, (DP $=6,39), \mathrm{CVp}$ de $56 \%$ e o intervalo de tempo predominante foi de 06 a 10 anos (27,27\%), seguido de 18 a 22 anos $(22,73 \%)$. A maioria $(45,45 \%)$ teve sua formação profissional na UFC, cuja escola de Enfermagem pertence à mesma instituição do Hospital Universitário Walter Cantídio, e o utiliza como campo natural de estágio. A titulação mais freqüente foi de especialista (77,27\%), seguida do título de mestre $(13,64 \%)$ e da graduação $(9,09 \%)$. A metade dos entrevistados ocupava cargo de chefia (50\%) e a outra metade não (50\%). A maioria tinha 02 empregos $(54,55 \%)$. Quanto à satisfação com a profissão, foi utilizada uma escala de 0 a 10 , para nortear as respostas, que corresponderam a valores acima ou iguais a sete da escala, conforme a seguir: $8(50 \%), 7(27,27 \%), 9(13,64 \%)$ e $10(9,09 \%)$. Portanto, pelos dados da tabela 1, evidenciamos que há no Hospital Universitário Walter Cantídio uma proporção considerável de enfermeiros especialistas, com nível de satisfação profissional bom, e com tempo de serviço a prestar na instituição. Entendemos que estes elementos podem constituir-se eixos potenciais e motivadores para a implementação de política que envolva a relação pesquisa ensino - assistência.

\section{O significado da palavra pesquisa}

O primeiro aspecto avaliado foi o que significa, para o enfermeiro, a palavra pesquisa, em seu sentido mais imediato. Destacamos as idéias dos entrevistados em duas categorias, apresentando parte do discurso:

- Categoria 1: Trabalho Científico - citada por 31,82\% dos entrevistados, que compreenderam a pesquisa como:

Investigação, ciência, descoberta (E10).

A busca pela verdade (E6).

Busca e novos conhecimentos (E2).

Descoberta, desafios (E8).

Metodologia (E22).

- Categoria 2: Melhora da Práxis/Aprimoramento/Crescimento - mencionada por $36,40 \%$ dos entrevistados; a pesquisa é destacada como uma atividade que impulsiona o crescimento, a reflexão e o aprimoramento da práxis.

Desenvolvimento de novas técnicas (E15).

Melhora do trabalho, aprimoramento dos conhecimentos, realização profissional (E17).

Crescimento profissional (E20).
Estes depoimentos estão bem próximos do que preconiza a literatura, ou seja, pesquisa é a capacidade de questionar, de reciclar, para que se possa atingir o patamar da produção própria, da capacidade de intervenção e de sua consolidação como algo absolutamente cotidiano. É a atitude de continuar aprendendo a aprender ${ }^{(2)}$.

Considerando os significados atribuídos, o enfermeiro do HUWC reconhece a importância e a necessidade de desenvolver pesquisa. Num hospital universitário ocorre um envolvimento diário com alunos e professores de várias disciplinas da Enfermagem e com outras áreas de conhecimento. Sendo assim, o hospital é um campo fértil para o desenvolvimento de novas técnicas, de busca de novos conhecimentos, aprimoramento e realização profissional.

\section{O significado da investigação científica}

Quanto ao significado de realizar investigação científica, obtivemos resultados animadores e, ao mesmo tempo, contundentes. Como atestamos nas seguintes categorias:

- Categoria 1: A pesquisa teorizando a prática - para 45,45\% dos entrevistados, a pesquisa traz soluções para o cotidiano, pois o enfermeiro a define como atividade que produz saber e que, por isso, deve estar associada à prática para a obtenção de mais conhecimentos, proporcionando, assim, respostas ao cotidiano de Enfermagem e novas aprendizagens.

Traz retomo ao serviço (E22).

... O trabalho de pesquisa tem que ser centrado no dia-adia... (E17).

Nós da enfermagem temos que crescer com a pesquisa, não podemos nos prender à parte técnica $(E 10)$.

Encontrar respostas para uma pergunta que você mesma fez ou que alguém fez (E11).

Estudo, dedicação, conhecimento (E7).

... Adquirir conhecimento (E2).

- Categoria 2: A pesquisa como tarefa laboriosa e pouco atrativa - para $36,40 \%$ dos entrevistados, a pesquisa é atividade laboriosa e pouco atrativa. É sentida pelos enfermeiros como algo árduo, cansativo, que requer tempo e que pressupõe um investimento pessoal muito grande.

Requer muito tempo (E16).

Muito cansativo (E12).

Muito difícil (E13).

Árduo (E6).

Complicado e difícil (E2).

Esses relatos encontram amparo na literatura, que define pesquisa como o cotidiano mais cotidiano, pois precisa condensar-se com expressões concretas do dia-a-dia. Sen- 
do assim, a teoria precisa confrontar-se com a prática, e a prática precisa retornar à teoria ${ }^{(2)}$. Entretanto, neste processo, a pesquisa ora pode acentuar mais prática, ora mais teoria.

\section{O gosto pela pesquisa}

Gostar de fazer pesquisa foi o terceiro aspecto avaliado, em relação à decisão do enfermeiro de pesquisar no HUWC, conforme apresentamos as categorias e os respectivos exemplos dos discursos dos entrevistados:

- Categoria 1: Gosto pela pesquisa - referida por $81,82 \%$ dos enfermeiros entrevistados, representa aqueles que gostam de fazer pesquisa e está associada ao valor que o enfermeiro atribui à investigação. Destacamos, entre as respostas da Cat. 1, as idéias do gosto pela investigação do trabalho científico, processo de elaboração de conhecimento em Enfermagem, incorporando novos conhecimentos ao cotidiano profissional e, também, por ser um marco diferencial entre quem pensa e quem faz. Alguns depoimentos são exemplificados, a seguir:

Tanto favorece o crescimento do profissional, quanto a instituição ganha com isso (E20).

Oportunidade de rever conceitos e aprofundar conhecimentos (E15).

A gente mostra o valor que tem em conhecimentos, se a gente tá pesquisando (E10).

Eu acredito que existem pessoas que nascem para executar tarefas, outras para pensarem (E11).

- Categoria 2: Desgosto pela pesquisa - referida por $18,18 \%$ dos entrevistados, representa aqueles que não gostam desta atividade, e está vinculada a não-superação das dificuldades encontradas pelo enfermeiro, dentre os quais apresentamos alguns depoimentos:

O tempo necessário é pouco (E21).

Não tenho muita oportunidade (E14).

Pouca experiência (E12).

Apesar de nossas dificuldades, a sociedade espera soluções, portanto, é preciso entrar em cena, a capacidade de dominar desafios concretos para planejar soluções adequadas, e paga para ter uma elite intelectual, que pode valer a pena, na medida das soluções apresentadas, pois ciência e tecnologia são as estratégias mais seguras da emancipação dos $\operatorname{povos}^{(2)}$.

Outro aspecto a ser considerado, sobre os depoimentos, é o preconceito com a ação, tido como antagônico ao pensamento. Ainda subsiste no espaço assistencial a idéia ultrapassada da divisão do trabalho entre os que pensam e os que fazem. Embora o modelo nightingaleano tenha sido o marco inaugural da Enfermagem moderna, instituindo a hierarquização do trabalho na profissão, ensejou com isso, a dicotomia entre trabalho manual (fazer) e trabalho intelectual (saber) na Enfermagem ${ }^{(11)}$. Esta estrutura de divisão do trabalho ainda tem raízes bem mais profundas, na Grécia antiga, onde se falava em dois tipos de artes. De um lado, as artes servis exercidas pelos servos e escravos que possuíam a característica profissional de serem ocupações manuais, e, de outro, as artes liberais, exercidas pelos homens livres, tidas como ocupações intelectuais ${ }^{(12)}$.

Sabemos que a sociedade latino-americana, por sua história colonial, reflete uma dicotomia entre o trabalho manual e o intelectual ${ }^{(13)}$, e ainda subsiste a concepção da Enfermagem como sendo uma profissão cujo conhecimento é estruturado numa atividade eminentemente prática; entretanto, há significativos movimentos de mudança desta concepção. Além disso, a prática de cuidar foi preparada, ao longo dos tempos, pela sociedade, como habilidade exclusiva ou predominantemente feminina e a prática de pesquisar, de criar conhecimentos, historicamente são concebidas como prática masculina ${ }^{(11)}$. Portanto, a concepção da Enfermagem empírica, estruturada no fazer, a qual se atualiza no cotidiano profissional é uma característica da dicotomia presente no processo de cuidar-investigar, que só poderá ser superada, se os enfermeiros pesquisarem enquanto administram os cuidados de enfermagem e cuidarem enquanto pesquisam ${ }^{(1)}$.

Tendo examinado o significado e afinidade com a pesquisa para os entrevistados, passamos a explorar fatores de sua formação profissional e situação atual no hospital universitário, entendendo-os também como elementos influenciadores em torno do tema pesquisa.

\section{$O$ reflexo do ensino acadêmico influenciando a capacidade de pesquisar}

A experiência vivenciada pelos enfermeiros como acadêmicos de enfermagem foi analisada em duas categorias, a seguir:

- Categoria 1: Insatisfação com o preparo teórico e metodológico recebido - representa o pensamento de $68,18 \%$ dos enfermeiros. Mesmo insatisfeitos com o desenvolvimento de suas potencialidades em relação à pesquisa, os entrevistados consideram haver recebido algum tipo de preparação, durante a vida acadêmica, direcionada para a prática de pesquisa. Eles destacam a importância de associar teoria, prática e pesquisa, desde as primeiras disciplinas de Enfermagem no âmbito hospitalar. Exemplificamos com os depoimentos:

Tive na cadeira de metodologia da pesquisa (E11).

Muito superficial. Só na época da monografia (E10).

Tive, mas em terceiro plano. A universidade só se baseava na teoria e prática (E21).

- Categoria 2: Faltando preparo teórico e metodológico representa a ausência total de preparo recebido pelos enfermeiros na graduação. Constitui $31,82 \%$ deles. 


\section{Não me lembro (E5).}

Na minha época não tinha essa formação acadêmica para a pesquisa (E1).

Estudo realizado aponta que a escola produz e inculca habitus específicos como o do cuidar mecanizado, fragmentado e descontextualizado. Portanto, é necessário que a escola desenvolva estratégias para estimular a realização de pesquisas e a consecução de novos conhecimentos, de modo a favorecer uma articulação entre os conteúdos teóricos e os saberes práticos ${ }^{(14)}$.

Uma política científica no âmbito acadêmico universitário, não mais centrada na ação técnica, que leve a redefinição dos espaços de produção científica e este contexto pressupõe a reforma curricular. A mudança mais sensível está em subjugar o currículo à pesquisa, com algumas dessas mudanças, iniciando na formação acadêmica básica, priorizando disciplinas de teoria e metodologia científica, que precisam aparecer nos primeiros semestres; substituir aulas expositivas por vídeos, liberando o professor para orientar a pesquisa dos alunos; montar práticas curriculares que se encaixem no desafio da própria pesquisa e reduzir ou extinguir a prova em favor do trabalho de pesquisa ${ }^{(2)}$.

As Diretrizes Curriculares Nacionais para os Cursos de Graduação em Enfermagem (DCENF), indicam as novas mudanças para a formação profissional da (o) enfermeira (o). Apontam, ainda para a necessidade de um corpo docente qualificado, com capacidade de desenvolvimento de pesquisa e extensão. Neste sentido, o Projeto Pedagógico dos Cursos de Enfermagem está redirecionado para estratégias que aproximam a formação acadêmica às necessidades locais de saúde e, consequentemente à consolidação do SUS, tendo como paradigma, as relações entre cultura, sociedade, saúde e educação(15).

Precisamos sair da óptica tecnicista para uma perspectiva mais ampla e crítica na Enfermagem. Um modo de ver que implique dimensões variadas: cognitivas, motoras e de atitudes; que permita maior autonomia, maior capacidade de resolver problemas, de adaptação às mudanças, de superação de conflitos, de comunicação, de trabalho em equipes, de decisão ética ${ }^{(16)}$.

Portanto, um profissional verdadeiramente comprometido com a sociedade é capaz de agir e refletir sobre sua realidade - um ser da práxis e não um ser passivo ${ }^{(13)}$.

\section{Os fatores limitantes e promotores da pesquisa no $\mathrm{HUWC}$}

Os resultados encontrados acerca dos fatores que facilitam ou que dificultam a pesquisa foram divididos nas categorias:

- Categoria 1: Enxergando dificuldades - compreendem $72,73 \%$ dos entrevistados, que apontaram somente dificuldades, conforme exemplificado nas cinco falas a seguir:
Falta de investimento na área da pesquisa (E10).

Falta ambiente para estudos dentro do hospital (E3).

Falta de computadores (E5).

Pouco incentivo por parte da Instituição (E8).

Falta bolsa de estudo (E16).

- Categoria 2: Enxergando facilidades - envolve 27,27\% dos entrevistados, que admitem a existência de facilidades, embora restritas, que motivam a pesquisa em enfermagem no HUWC, conforme exemplificam as quatro falas abaixo:

São restritas (E19).

O querer realizá-las, estar dentro de um hospital escola (E5).

A gente faz pesquisa porque tem vontade de crescer (E10).

Os dados permitem-nos constatar os desafios a serem ultrapassados pelo enfermeiro, quando se trata de fazer pesquisa em um hospital-universitário e público.

O desenvolvimento da Enfermagem ao longo dos anos nos mostra uma trajetória de lutas por espaço e reconhecimento profissional. Apesar disso, muitas foram e ainda são as dificuldades enfrentadas pela profissão no que tange à formulação de um saber específico que confira cientificidade às suas ações e visibilidade social ${ }^{(11)}$.

Podemos constatar que dificuldades e facilidades enfrentadas e identificadas no estudo são meras repetições de outras realidades brasileiras, quando se trata de se fazer pesquisa na Enfermagem, tais como, a baixa valorização dada à pesquisa; pouco ou nenhum incentivo das instituições, reduzido conhecimento de métodos ou estratégias para a prática de pesquisar, escassez de tempo, de recursos humanos e materiais; a maioria dos enfermeiros não encontra tempo para leituras e ter que desenvolver estudos, por sua jornada em mais de uma instituição ${ }^{(11-13)}$.

Os fatores que interferem a pesquisa pelos enfermeiros, no HUWC, foram distribuídos em fatores limitantes e promotores, conforme a seguir:

1. Fatores limitantes: falta de tempo no local de trabalho; de uma política institucional de incentivo à pesquisa; de apoio financeiro; de recursos humanos e materiais; de local adequado para estudos; definição de uma linha de pesquisa na instituição; da política de valorização para o enfermeiro que deseja cursar mestrado e doutorado; de esclarecimento adequado aos pesquisadores do Comitê de Ética do complexo hospitalar, que compreendam os objetos de estudo da Enfermagem; de integração com estagiários; supremacia da prática de cuidar em detrimento da prática de pesquisar; trabalhar em mais de uma instituição; extensa carga horária de trabalho; desconhecimento teórico-metodológico sobre pesquisa. 
2. Fatores promotores: a motivação e o esforço individual; o fato de trabalhar em um hospital-escola; de ter fácil acesso aos pacientes e de possuir colegas professores.

\section{CONSIDERACÕES FINAIS}

Este estudo mostra que a semântica da palavra pesquisa e o ato de desenvolvê-la estão bem definidos e ancorados na prática profissional dos enfermeiros; com significados que apontam para a importância da pesquisa quanto à busca da verdade, de novos conhecimentos, da identificação de problemas e respostas para o próprio cotidiano de Enfermagem, sendo este o foco privilegiado para as pesquisas, e estas, fonte de realização e crescimento pessoal e profissional. Sendo assim, para ultrapassar a ação meramente técnica, é necessário que a pesquisa na Enfermagem seja aplicada no dia-a-dia, ou seja, no ensino, na promoção da saúde e na qualidade de vida à população atendida.

Apesar de o HUWC ser um ambiente favorável para a realização de pesquisas, um campo vasto, um laboratório em potencial, e que, por sua vez, abriga no seu cotidiano a trilogia ensino/pesquisa/assistência, isto se torna um paradoxo frente às dificuldades encontradas para desenvolvê-las.

A quase-totalidade dos enfermeiros gosta de fazer pesquisa, segundo os depoimentos dos sujeitos investigados.

\section{REFERÊNCIAS}

1. Rozendo CA, Coller N. Interface cuidado-investigação em enfermagem. Rev Gaúcha Enferm. 1999;20(2):28-36.

2. Demo P. Desafios modernos da educação. Petrópolis: Vozes; 2000.

3. Brasil. Ministério da Saúde. Secretaria de Gestão de Investimento em Saúde. Formação pedagógica em educação profissional na área de saúde: enfermagem núcleo estrutural: proposta pedagógica: campo da ação. Módulo 5. $2^{\mathrm{a}}$ ed. Brasília; 2003. (Série F. Comunicação e Educação em Saúde)

4. Echer IC, Crossetti MGO, Paskulin LMG, Cogo ALP, Santos VBD, Sottomaior VS, et al. Criação da Comissão de Pesquisa do Grupo de Enfermagem do Hospital de Clínicas de Porto Alegre. Rev Gaúcha Enferm. 1998;19(1):56-9.

5. Vale EG. Discurso da presidente da ABEN Nacional. In: Anais do $10^{\circ}$ Seminário Nacional de Pesquisa em Enfermagem: a interdependência do cuidar e do pesquisar na enfermagem; 1999; Gramado, BR. Porto Alegre: ABEn-RS; 1999. p. 7-8.

6. Amorim MHC, Bringuente MEO, Castro DSC. Relatando experiências e repensando a pesquisa em enfermagem. Anais do $10^{\circ}$ Seminário Nacional de Pesquisa em Enfermagem: a interdependência do cuidar e do pesquisar na enfermagem; 1999; Gramado, BR. Porto Alegre: ABEn-RS; 1999. p. 55-8.
Esta prática aparece em quase todas as falas como um ganho pessoal e institucional e de valorização pessoal, mas que depende apenas de investimento e esforço próprios, ou seja, não há uma política institucional e, por isso, se torna uma atividade laboriosa, árdua, desmotivando aqueles que não conseguem superar as dificuldades individuais.

Portanto, o que se observa em nossa realidade é a supremacia do ato de cuidar em detrimento da prática de pesquisar. Entretanto, sabemos que a missão do HUWC segue como estratégia a ordem - pesquisa, ensino e assistência - não devendo existir o prejuízo de uma das partes em relação às outras. Isto significa dizer que não é a quantidade de atendimentos que fazemos o mais importante, mas sim a qualidade deste atendimento.

Se pensarmos na integralidade do cuidado hospitalar, tal abordagem poderia implicar em garantir desde a criação de um ambiente que resultasse em conforto e segurança para a pessoa hospitalizada até o consumo de todas as tecnologias de saúde disponíveis, como aquelas ligadas a equipamentos, procedimentos e a saberes bem estruturados ${ }^{(17)}$.

Acreditamos que, apesar da escassez de recursos humanos, físicos, materiais e financeiros de nossa instituição de saúde, é preciso partir das possibilidades atuais. Enfim, fazer pesquisa implica sempre ousadia e obstáculos a serem transpostos. E é bom que existam mesmo, pois só assim estaremos sempre nos superando.
7. Minayo MCS, Deslandes SF, Cruz Neto O, Gomes R. Pesquisa social: teoria, método e criatividade. $6^{\mathrm{a}}$ ed. Petrópolis: Vozes; 1996.

8. Polit DF, Hunler B. Fundamentos de pesquisa em enfermagem. $3^{\text {a }}$ ed. Porto Alegre: Artes Médicas; 1995.

9. Minayo MCS. Pesquisa social. 20ª ed. Petrópolis: Vozes; 2002.

10. Conselho Nacional de Saúde. Resolução n. 196, de 10 de outubro de 1996. Dispõe sobre diretrizes e normas regulamentadoras de pesquisas envolvendo seres humanos [legislação na Internet]. Brasília; 1996 [citado 2005 dez. 12]. Disponível em: http:// conselho.saude.gov.br/docs/Resolucoes/Reso196.doc.

11. Brasil. Ministério da Saúde. Secretaria de Gestão de Investimento em Saúde. Formação pedagógica em educação profissional na área de saúde: enfermagem núcleo integrador: vivenciando uma ação docente autônoma e significativa na educação profissional em enfermagem. Módulo 11. $2^{\mathrm{a}}$ ed. Brasília; 2003. (Série F. Comunicação e Educação em Saúde).

12. Brasil. Ministério da Saúde. Secretaria de Gestão de Investimento em Saúde. Formação pedagógica em educação profissional na área de saúde: enfermagem núcleo estrutural: proposta pedagógica: o plano de ação. Módulo 7. 2a ed. Brasília; 2003. (Série F. Comunicação e Educação em Saúde). 
13. Elsen I. A pesquisa em enfermagem: como anda este processo na região sul? In: Anais do $7^{\circ}$ Seminário Nacional de Pesquisa em Enfermagem: pesquisa, ensino e assistência: o desafio profissional; 1994; Fortaleza, BR. Fortaleza: ABEn-CE; 1994. p. 35-6.

14. Daher DV, Santos FHE, Escudeiro CL. Cuidar e pesquisar: práticas complementares ou excludentes. Rev Lat Am Enferm. 2002;10(2):145-50.

15. Fernandes JD, Xavier IM, Ceribelli MIPF, Bianco MHC, Maeda D, Rodrigues MVC. Diretrizes curriculares e estratégias para implantação de uma nova proposta pedagógica. Rev Esc Enferm USP. 2005;39(4):443-9.
16. Freire P. Educação e mudança. $20^{\mathrm{a}}$ ed. São Paulo: Paz e Terra; 1994.

17. Cecílio LCO, Merhy EE. A integralidade do cuidado como eixo da gestão hospitalar. In: Pinheiro R, Mattos RA. Construção da integralidade: cotidiano, saberes e práticas em saúde. Rio de Janeiro: IMS; 2003. p. 197-210.

\section{Agradecimentos}

Ao Vianney Mesquita, Membro-Titular (cadeira n ${ }^{\circ}$ 37) da Academia Cearense da Língua Portuguesa e professor Adjunto do Centro de Humanidades da UFC, pela revisão ortográfica.

À Nara Lígia Gregório Fiúza, acadêmica de Enfermagem do curso de Graduação da Faculdade de Farmácia, Odontologia e Enfermagem FFOE/UFC, por sua colaboração na fase de realização e transcrição das entrevistas. 